\title{
Umbilical Catheter Complications in Newborns during Prone Position: A Pilot Study
}

\author{
Inge Arnts ${ }^{1,2}{ }^{*}$, Ninke Schrijvers ${ }^{1}$, Coranne Meester $^{1}$, Joannes Groenewoud ${ }^{3}$, \\ Kian Djien Liem 1 \\ ${ }^{1}$ Department of Neonatology, Radboud University Nijmegen Medical Centre, Nijmegen, The Netherlands \\ ${ }^{2}$ Department of Pain and Palliative Care, Nijmegen, The Netherlands \\ ${ }^{3}$ Department of Health Evidence, Radboud University Nijmegen Medical Centre, Nijmegen, The Netherlands \\ Email: ${ }^{*}$ Inge.Arnts@radboudumc.nl
}

Received 11 October 2014; revised 10 November 2014; accepted 22 November 2014

Academic Editor: Amy F. Schaeffer, Shepherd University, United States

Copyright (C) 2014 by authors and Scientific Research Publishing Inc.

This work is licensed under the Creative Commons Attribution International License (CC BY). http://creativecommons.org/licenses/by/4.0/

c) (i) Open Access

\begin{abstract}
Introduction: It is not known whether prone position of newborns with umbilical catheters increases the complication risk. Purpose: Analysing complications of umbilical catheters in newborns during prone positioning and analysing if local complications as a wet or red rim increase severe complications. Subjects: Newborns $(<24$ weeks -42 weeks gestational age) with an umbilical catheter, inserted on our third level 17-bed neonatal care unit. Design: A prospective observational pilot study (during September 2009-September 2010). Methods: Each neonate was positioned for at least 12 hours in 48 consecutive hours in the prone position. Results: 88 newborns were included. Central line associated bloodstream infection was the main reason for catheter removal due to complications. A wet or red umbilical rim did not increase the major complications. The relative risk for central line associated bloodstream infection in case of pus excretion was 3.3 as compared with clean umbilical stump $(p=0.015)$. There were no complications as excessive blood loss or obstructions. Conclusion: An umbilical catheter should not be considered as a contraindication for prone positioning. In case of wet rim or pus excretion from the umbilical stump, removal of the catheter should be seriously considered.
\end{abstract}

\section{Keywords}

Umbilical Venous Catheters, Umbilical Arterial Catheters, Complications, Nursing Care, Prone Position, Newborns

\footnotetext{
${ }^{*}$ Corresponding author.
}

How to cite this paper: Arnts, I., Schrijvers, N., Meester, C., Groenewoud, J. and Liem, K.D. (2014) Umbilical Catheter Complications in Newborns during Prone Position: A Pilot Study. Open Journal of Nursing, 4, 859-867. 


\section{Introduction}

The placement of umbilical catheters (UCs) in Neonatal Intensive Care Units (NICUs) is a common procedure. In the first two days after birth, the umbilical vein and the umbilical arteries can be used for the insertion of venous and arterial catheters. An umbilical central venous catheter (UVC) is required to administer fluids, medications and parenteral nutrition to critically ill newborns. An umbilical arterial catheter (UAC) is used in critically ill newborns when frequent blood sampling is necessary or when continuous blood pressure monitoring is required. The use of UCs is not without risks. Several complications (in a range of $2.5 \%-20 \%$ ) of UVCs have been described in the literature, such as central line associated bloodstream infection (CLABSI), thrombosis, dislocation and extravasation [1]-[6].

For UACs, complication rates are reported from 5.5\% to $32 \%$, including complications such as vascular spasm, ischemia, haemorrhage, CLABSI and thrombosis [7] [8]. Frequent observation of the position of the UC in the umbilical stump has been proposed as a measure to prevent catheter complications such as dislocation, which can result in leakage and blood loss. For these reasons, in our NICU, the prone positioning of a neonate with an UC is not allowed. We assume that prone positioning will increase the risk of local complications too, such as a persistent wet umbilical stump or red umbilical rim. This might be a risk factor for developing major complications, such as dislocation and CLABSI. However, this presents a dilemma because prone positioning is assumed to be the best position for these vulnerable patients with respiratory problems [9] [10]. In a questionnaire completed by 10 Dutch NICUs, prone positioning of a neonate with an UC is a common practice in 7 NICUs because of the respiratory advantage (unpublished data). However, they never analysed the UC complication rate in prone position. In their experience, there was no increasing risk of severe UC-related complications when positioning a neonate with an UC in the prone position. In the literature, no study has specifically described the complications associated with the prone positioning of newborns with an indwelling UC. In the guidelines from the Centers for Disease Control, no recommendation has been made for positioning of newborns with an UC [11].

Because prone position with an indwelling UC is a common practice in most NICUs due to its respiratory advantage for the neonate, we decided to change our policy. From the start of this study it was allowed to position newborns with an indwelling UC in prone position if possible. Simultaneously, we started a prospective observational pilot study on UC complications in these newborns with UC in situ during prone position. An approval of the medical committee of the institution or informed consent is not necessary because this observation study is based on common practice in most Dutch NICUs.

\section{Purpose}

The primary aim of this study was to analyse the incidence of UC complications leading to catheter removal when newborns with UCs were positioned in the prone position at least 12 hours in 48 sequential hours. The secondary aim was to analyse whether local complications would increase the risk for major complications.

\section{Sulbjects}

The setting was a third level 17-bed NICU with an average of 500 admissions per year.

In this prospective observational pilot study, we collected data from all newborns (from 24 weeks gestational age until term) admitted to the NICU who received an UC and nursing care in the prone position. Since prone position is not always continuously applied, only participants with at least 12 hours ( 3 times of 4 consecutive hours) during 48 consecutive hours in the prone position were included. Newborns who died or were transferred to another hospital with an indwelling UC for at least 48 hours and who met the criteria were also included because the effect of a minimum of 12 hours in the prone position and the possible development of local or major complications was the primary and most important goal of our study.

Excluded were: 1) newborns who did not meet the inclusion criteria of a minimum of 12 hours in the prone position over 48 consecutive hours; 2) newborns with severe persistent pulmonary hypertension; 3) newborns with abdominal surgical problems; 4) newborns who underwent Extra Corporeal Membrane Oxygenation (ECMO) treatment; 5) newborns with an UC inserted in another centre; 6) newborns with a wet and/or red umbilical stump and rim within 12 hours after insertion of the UC. 


\section{Design and Method}

Over a 12-month period (during September 2009-September 2010), data was collect by the investigators.

Before this pilot study, all nurses were instructed in the standard care of an UC, the inclusion and exclusion criteria, the use of the checklist (Table 1) and in recognizing the complications. Prone positioning was not allowed in the first 12 hours to ensure proper observation of leakage or blood loss. Prone positioning was allowed when the umbilical stump and the rim were dry and without redness. Thus, twelve hours after insertion of the UC, the nurse initiated prone positioning when possible.

During the total observation period, each neonate was positioned for at least 12 hours of 48 consecutive hours in the prone position. Endpoints of the analysis were removal of the UC because of end of treatment or complications, transfer to another hospital or newborns who died with an indwelling UC $>2$ days.

Clinical characteristics, complications, indwelling time of the catheter, hours in prone position, observation of the umbilical stump, occlusion of the infusion pump and the reason for removal were recorded every two hours by the nurses using a standard manual of criteria. Reasons for removal of the UC can be divided in two groups: elective reasons (end of therapy, discharge and death) and non-elective reasons. Non-elective reasons included obstruction of the UC (difficult or impossible to flush, inability to administer fluid in 3 seconds), leakage/blood loss (around the UC insertion site), dislocation and CLABSI. Local complications not directly resulting in removal included redness of the skin rim of the umbilical stump or a moist or wet umbilical stump.

In this study we used the CLABSI definition of health care-associated infection and criteria for specific types of infection in acute setting as mentioned for patients $<1$ year in the Centers for Disease Control and Prevention (CDC) [12].

CLABSI is a laboratory-confirmed bloodstream infection with a UVC and/or UAC in place for a minimum of two days or in place on the day of event or the day before.

Laboratory-confirmed CLABSI was defined by using one of the first two following definitions:

Table 1. Nursing observation sheet: prone position with an indwelling UC.

\footnotetext{
Position Prone/Supine/Lateral

Umbilical stump

Wet/Dry

Clear/Blood/Pus/Other

Position UC in $\mathrm{cm}$

UC rim

Wet/Dry

Release of fixation

Heart rate/Respiration rate

$\mathrm{O}_{2}$ saturation

Inspiration fraction of $\mathrm{O}_{2}$

Respiration support

Tidal volume

Pip/Peep at $12 \mathrm{~h}$

Occlusion alarm

Clinical signs

Antibiotics

Others
}

Fixation

Parameters

Infusion

Infection 
Criterion 1: one or more positive blood cultures (with the exception of skin micro-organism) not related to another infection source.

Criterion 2: clinical signs of sepsis (especially for patients $<1$ year: fever or hypothermia, apnea or bradycardia), two or more positive blood cultures drawn on separate occasions with the same micro-organism (incl. skin micro-organism) and no other infection source. Criterion elements must occur within a timeframe that does not exceed a gap of 1 day.

An important and in practice common used criterion, but not mentioned in the CDC, is the criterion of only clinical sepsis (CSEP). In this special patient group, clinical signs are frequently the only manifestation of sepsis in the beginning and this is usually sufficient enough to start treatment with antibiotics. Sometimes it is very hard to obtain a blood culture of the small blood vessels, which frequently results in insufficient outcome for sensitive detection of bacteraemia [13]. Because of this reason, we added "CSEP" as an additional CLABSI definition. This includes clinical signs of sepsis as mentioned in criterion 2, no or one positive blood culture (only skin micro-organism), and no other infection source other than a CVC (in situ or removed in 24 hours), and medical reasons to start sepsis treatment. This is comparable with other studies with their specific description of CSEP [14] [15].

Because in many studies only the laboratory-conformed CLABSI as mentioned by the CDC is followed, we decided to use the laboratory-confirmed CLABSI and the CSEP separately.

Thrombosis screening was only indicated when there was a clinical reason for thrombosis.

\section{Material and Procedure}

An UCC of UAC (with respectively charrières 5 and 3.5, Kendall Argyle, Tyco Healthcare, Tullamore, Ireland) was inserted under sterile conditions by trained neonatologists, nurse practitioners and residents who followed a standard protocol outlining the insertion procedure. In most cases, UAC and UVC were inserted at the same time. The tip of the UAC was positioned above the aortic bifurcation and below the origins of the renal arteries (under the 2nd lumbar vertebrae). The tip of the UVC was positioned in the inferior vena cava just before the junction with the right atrium and at the level of diaphragm. After placement of the UC, the position of the tip was verified by X-ray and repositioned if necessary before parenteral nutrition or drugs were administered. The catheter was fixated with a suture through the umbilical jelly. A second fixation of the catheter with plaster (a neo-bridge) was used for extra safety [16]. Twice a day, the insertion place was disinfected with a $0.5 \%$ chlorhexidine/alcohol $70 \%$ solution. In order to reduce the UC complications, especially CLABSI, our protocol prescribed that when possible an UC should be removed as soon as possible.

As a standard of care, heparin was continuously administered at a dose of two units per hour. Only saline with heparin was administered through the UAC. For the UVC, all medications and fluids could be used, except for blood products. The entire drip system of the UVC and UAC was replaced every 96 hours and 48 hours, respectively, according to our hospital's protocol. Drip systems for the administration of intralipid (fat solution) were changed every 24 hours. The catheter insertion site was checked every two hours for signs of inflammation, leakage and blood loss as part of the standard of care.

All 60 NICU nurses were competent in the care of UCs. During the primary education of NICU nurses, the nurses were trained and assessed in the care of an UC. The nurses were re-trained every three years. The standard care for a neonate in the supine or prone positioning did not differ. All of the patients were monitored for heart rate, respiratory rate and arterial oxygen saturation using pulse oximetry.

\section{Mean Outcome Measures}

The mean and standard deviation were used to describe continuous variables, which followed a normal distribution.

To check for statistical significance between categorical variables, Pearson's chi-square test or Fisher's exacttest (in case one or more cells had an expected count less than five) was used. The relative risk was used as the association measure between a dichotomous outcome and a dichotomous risk factor. The data are presented with $95 \%$ confidence intervals. A confidence interval not containing the value 1 indicated a significant relationship between the risk factor and the outcome. Effects were considered statistically significant at the 0.05 threshold ( $\alpha$ $=0.05)$. SPSS 20.0 for Windows was used for statistical analysis. 


\section{Results}

Hundred six patients were selected. Eighteen patients were excluded because they did not meet the inclusion criteria of the minimum hours in the prone position. Finally, 88 patients were included. All these newborns had an UVC (57\% single lumen, $43 \%$ double lumen). In $44.3 \%$ cases an UAC was inserted in the same time. The mean birth weight was 1345 gram ( $\min 400$ gram, max 4220 gram). The mean gestational age was 29.6 weeks (min. 24 weeks, max. 40.0 weeks, Table 2). The main reason for insertion of an UC was the need for total parenteral feeding. There was a wide range of hours in the prone position (range $14 \mathrm{~h}-279 \mathrm{~h}$ ) during this study with a mean of 80.5 hours. The mean indwelling time for the UC was 6 days (mean 145 hours, range 24 hours 336 hours) with a total of 529 catheter days.

The main reason for catheter removal was elective removal because end of treatment (Table 3). There was no excessive blood loss or perforation of the catheter. There was a low incidence of dislocation, followed by leakage and obstruction. No thrombosis screening was indicated during this study, based on clinical signs. There were no specific occlusion alarms on the infusion pump.

CLABSI (laboratory-confirmed CLABSI and CSEP) was the most important complication to remove a catheter $(18.2 \%, 30.2$ per 1000 catheter days).

However, laboratory-confirmed CLABSI was only confirmed in $9.1 \%$ of the CLABSI removals (17 CLABSIs per 1000 catheter days). Only $4.5 \%$ met criterion 1 and $4.5 \%$ met criterion 2 for laboratory-confirmed CLABSI.

Coagulase negative staphylococcus was the most important and common cause of laboratory-confirmed CLABSI.

There was a high incidence of local complications (redness of the umbilical rim 13.6\%, persistent wet or moist umbilical stump $64.8 \%$, pus excretion $9.1 \%$, Table 4). Almost all umbilical stumps with a red rim had a persistent wet or moist umbilical stump.

The incidence of CLABSI (laboratory-confirmed CLABSI and CSEP) in a persistent wet umbilical was $17.9 \%$ versus $19.4 \%$ in the dry umbilical stump. This means that the relative risk for CLABSI in case of a persistent wet umbilical stump was 0.9 as compared to a dry rim $(95 \%$ confidence interval $0.38-2.4, p=0.91)$. The relative risk for only laboratory-confirmed CLABSI in case of a wet umbilical stump compared to dry umbilical rim equals $0.6(95 \%$ confidence interval $0.15-2.1, \mathrm{p}=0.4)$.

There was also no increased relative risk in case of redness of the umbilical rim $(\mathrm{p}=0.34)$.

In 8 cases (9.1\%), we found pus excretion around the stump. In 3 cases, the UCs were electively removed and in one case the UC was removed because of leakage. In these 4 cases there were no clinical signs and no CLABSI developed within a timeframe of 24 hours. Only in 4 cases there was CLABSI ( 2 numbers laboratoryconfirmed CLABSI and 2 numbers CSEP) with pus excretion. The relative risk for CLABSI in case of pus excretion was 3.3 as compared to clean umbilical stump $(95 \%$ confidence interval $1.40-7.93, p=0.014)$. The relative risk for only laboratory-confirmed CLABSI in cases of pus excretion compared to clean umbilical stump was also 3.3 ( $95 \%$ confidence interval $0.80-13.87, \mathrm{p}=0.1)$.

In 7 cases of pus excretion, the umbilical surrounding was also defined as wet or moisty.

Only in one case there was also a red rim. The relative risk for pus excretion in case of a wet umbilical stump compared to a dry rim equals $4(95 \%$ confidence interval $0.515-31.06, p=0.14)$. However this is not significant, we assume the possibility that a wet rim will increase the risk of pus excretion.

A double lumen UVC or a simultaneous insertion of UVC and UAC did not increase the relative risk for CLABSI (laboratory-confirmed CLABSI and CSEP). This was respectively $\mathrm{p}=0.24$ and $\mathrm{p}=0.48$.

Also birth weight and gestational age did not influence the complication risks in this study (both $\mathrm{p}>0.5$ ).

Because the duration of hours in prone position is depending on the indwelling time of the UC, a relative risk of complications only depending on hours in prone position cannot be made.

\section{Discussion}

In this observational pilot study, no excessive blood loss, occlusion or dislocation was seen during the prone position period. There was a relatively high incidence of CLABSI (using laboratory-confirmed CLABSI and CSEP). However, when using only the laboratory-confirmed CLABSI there is still a high CLABSI incidence (9\%, 17 CLABSI per 1000 central line days), compared to the literature (2.3 - 17 CLABSI per 1000 central line days) [2] [17]-[21]. Because there is no comparable study, we cannot conclude whether this high incidence is especially associated because of the prone position. However, this can be a starting point for further research. 
Table 2. Baseline characteristics.

\begin{tabular}{cc}
\hline & Patients \\
$\mathrm{N}=88$ & $39(44.3 \%)$ \\
UAC & $88(100 \%)$ \\
Double lumen UVC & $38(43 \%)$ \\
Birth weight (grams) mean \pm SD & $1345 \pm 6665$ \\
Gestational age (weeks) mean \pm SD & $29.7 \pm 3.0$ \\
Indwelling time (days) mean \pm SD & $6.0 \pm 2.8$ \\
\hline
\end{tabular}

UAC: Umbilical Arterial Catheter; UVC: Umbilical Venous Catheter.

Table 3. Reasons for removal.

\begin{tabular}{cc}
\hline & Patients \\
$\mathrm{N}=88$ & $16(18.2 \%)$ \\
CLABoratory-confirmed CLABSI & $8(9.1 \%)$ \\
CSEP & $8(9.1 \%)$ \\
Obstruction & $1(1.1 \%)$ \\
Leakage & $1(1.1 \%)$ \\
Dislocation & $5(5.7 \%)$ \\
Perforation & 0 \\
Death/Discharge & $14(15.9 \%)$ \\
Elective removal/ end of treatment & $51(58.0 \%)$
\end{tabular}

Total catheter days: 529; CLABSI: central line associated bloodstream infection; CSEP: clinical sepsis.

Table 4. Umbilical conditions.

\begin{tabular}{cccc} 
& $\begin{array}{c}\text { Patients } \\
\mathrm{N}=88\end{array}$ & $\begin{array}{c}\text { CLABSI (Total) } \\
\mathrm{N}=16\end{array}$ & $\mathrm{p}$-value \\
\hline Red rim & $12(13.6 \%)$ & & $\mathrm{ns}$ \\
Wet or moisty rim & $57(64.8 \%)$ & $10(17.9 \%)$ & $\mathrm{ns}$ \\
Pus excretion & $8(9.1)$ & $4(50 \%)$ & 0.014 \\
Dry rim & $11(12.5 \%)$ & $2(19.4 \%)$ & $\mathrm{ns}$ \\
\hline
\end{tabular}

CLABSI: central line associated bloodstream infection.

A comparison between the studies is difficult because different CLABSI definitions have been used. Probably this can declare our high CLABSI incidence too. In $50 \%$ of our defined CLABSIs there were laboratory-confirmed CLABSIs according to the CDC criterion.

Well-known factors that can increase CLABSI are indwelling time of an UC, gestational age and total parenteral feeding [2] [11] [19] [22] [23].

Gestational age did not increase the risk of complications in this study. Total parenteral feeding was the main reason for UC insertion. The need of total parenteral feeding consists in a longer indwelling time of a UVC, which probably influences the outcome (reasons for removal). The maximum indwelling time of the UVC in our study did not exceed 14 days, as described in more studies, which is relatively safe [2] [11] [22].

Chien et al. (2002) found an adjusted relative risk from 2.5 for the use of an UC comparing to no UC use, causing nosocomial bloodstream infections [22]. Also frequent manipulations of the UC are a contributing factor in CLABSI. An average of 3.2 manipulations per day $(0-15)$ is associated with a 5 -fold increase in CLABSI in 
very low birth weight infants. We did not count the manipulations per day, so this adjusted risk cannot be mentioned, but is an important issue.

We could not find data about the incidence of local complications for UCs, such as redness of the umbilical rim or a persistent wet umbilical stump in the literature. Therefore we assumed that the incidence of these local complications is very low or negligible. However, in this study we found a high incidence of these local complications. A reason for this high incidence might be the high humidity around the umbilical stump during prone position. Despite this high incidence, a persistent wet umbilical stump and redness of the umbilical rim were not a risk factor for major complications, such as leakage, dislocation or CLABSI.

On the other hand, a wet umbilical stump increased the risk of pus excretion (although not significant) and pus excretion increased the risk of CLABSI significantly. Thus indirectly, a wet rim can possibly increase CLABSI. In our previous study (2005-2006) in were complications in peripherally inserted central venous catheters and umbilical venous catheters were analysed, there was a CLABSI incidence (including laboratoryconfirmed CLABSI and CSEP) in UVCs of $15 \%$ [23]. This outcome is comparable with the CLABSI incidence in this study. However the laboratory-confirmed CLABSI in our previous study is lower (4.3\%). Although both studies have been conducted on the same ward, again we cannot make a reliable comparison while the first study was a retrospective observational study with a larger population.

This study has an important limitation. It is not a randomized study, but only an observational cohort study. The duration of prone position is also not standardized. Therefore, we consider this study as a pilot study in order to get some impression about the magnitude of the complications of UC during prone position, because there is a lack of data about it in the literature. A larger prospective randomized controlled trial of UC comparing prone and supine position can be recommended.

\section{Conclusions}

The application of prone positioning in combination with umbilical catheters in newborn infants did not result in more severe complications as compared with the literature data of UC in supine position. However, the laboratory-confirmed CLABSI incidence is relatively high. There is a high incidence of local umbilical complications without increasing the risk of major complications with exception of pus excretion from the umbilical stump. In case of a wet rim or pus excretion around the umbilical stump, removal of the UC should be seriously considered.

So, an umbilical catheter should not be considered as a contraindication for prone positioning when frequent observation of the umbilical rim is a standard care. A larger prospective randomized controlled trial is recommended to improve support for this conclusion.

\section{Conflict of Interest}

The authors have declared no conflict of interest.

\section{Funding}

This research received no specific grant from any funding agency in the public, commercial, or not-for-profit sectors.

\section{Acknowledgements}

We thank American Journal experts for their linguistic support.

We thank also the nursing infusion group and Mrs. P. Mansvelt, Mr. Th. Peeters, Mrs A. Heijnen, Mrs. K. Frank and Dr. A.van Heijst for their support during the realisation of this study and data analysis.

\section{References}

[1] Bradshaw, W.T. and Furdon, S.A. (2006) A Nurse's Guide to Early Detection of Umbilical Venous Catheter Complications in Infants. Advances in Neonatal Care, 6, 127-138. http://dx.doi.org/10.1016/j.adnc.2006.03.002

[2] Butler-O’Hara, M., Buzzard, C.J., Reubens, L., McDermott, M.P., DiGrazio, W. and D’Angio, C.T. (2006) A Randomized Trial Comparing Long-Term and Short-Term Use of Umbilical Venous Catheters in Premature Infants with Birth Weights of Less Than 1251 Grams. Pediatrics, 118, e25-e35. http://dx.doi.org/10.1542/peds.2005-1880 
[3] Revel-Vilk, S. and Ergaz, Z. (2011) Diagnosis and Management of Central-Line Associated Thrombosis in Newborns and Infants. Seminars in Fetal \& Neonatal Medicine, 16, 340-344.

[4] Salonvaara, M., Riikonen, P., Kekomäki, R. and Heinonen, K. (1999) Clinically Symptomatic Central Venous Catheter-Related Deep Venous Thrombosis in Newborns. Acta Paediatrica, 88, 642-646. http://dx.doi.org/10.1111/j.1651-2227.1999.tb00014.x

[5] Sheridan, R.L. and Weber, J.M. (2006) Mechanical and Infectious Complications of Central Venous Cannulation in Children: Lessons Learned from a 10-Year Experience Placing More Than 1000 Catheters. Journal of Burn Care \& Research, 27, 713-718.

[6] Veldman, A., Nold, M.F. and Michel-Behnke, I. (2008) Thrombosis in the Critically Ill Neonate: Incidence, Diagnosis and Management. Vascular Health and Risk Management, 4, 1337-1348.

[7] Furdon, S.A., Horgan, M.J., Bradshaw, W.T. and Clark, D.A. (2006) Nurses' Guide to Early Detection of Umbilical Arterial Catheter Complications in Infants. Advances in Neonatal Care, 6, 242-256. http://dx.doi.org/10.1016/j.adnc.2006.06.001

[8] Lin, S.J., Kolz, P.F., Davis, W. and Vicari, F. (2009) Lower Extremity Ischemia Following Umbilical Artery Catheterization: A Case Study and Clinical Update. International Journal of Surgery, 7, 182-186.

[9] Balaguer, A., Escribano, J., RoquéiFiguls, M. and Rivas-Fernandez, M. (2013) Infants Position in Newborns Receiving Mechanical Ventilation. Cochrane Database of Systematic Reviews, 28, 3. http://dx.doi.org/10.1002/14651858.CD003668

[10] Sahni, R., Saluja, D., Schulze, K.F., Kashyap, S., Ohira-Kist, K., Fifer, W.P. and Myers, M.M. (2003) Quality of Diet, Body Position and Time after Feeding Influence Behavioral States in Low Birth Weight Infants. Pediatric Research, 52, 399-404. http://dx.doi.org/10.1016/j.adnc.2006.06.001

[11] O’Grady, N.P. Alexander, M., Burns, L.A., Dellinger, E.P., Garland, J., Heard, S.O., Lipsett, P.A., Masur, H., Mermel, L.A., Pearson, M.L., Raad, I.I., Randolph, A.G., Rupp, M.E. and Saint, S., Centres for Disease Control and Prevention, Healthcare Infection Control Practices Advisory Committee (2011) Guidelines for the Prevention of Intravascular Catheter-Related Infections. American Journal of Infection Control, 39, S1-S34.

[12] Centers for Disease Control and Prevention/National Healthcare Safety Network. Protocol correction, clarifications and additions; device associated module CLABSI. This protocol has not yet been added to the current posted NHSN protocols. January 2014.

[13] Schelonka, R.L., Chai, M.K., Yoder, B.A., Hensley, R.M., Brockett, R.M. and Ascher, D.P. (1996) Volume of Blood Required to Detect Common Neonatal Pathogens. The Journal of Pediatrics, 129, 275-278. http://dx.doi.org/10.1016/S0022-3476(96)70254-8

[14] Van der Zwet, W.C., Kaiser, A.M., van R.M., E., Berkhof, J., Fetter, W.P.F., Parlevleit, G.A. and Vandenbroucke, C.M.J.E. (2005) Nosocomial Infections in a Dutch Neonatal Intensive Care Unit: Surveillance Study with Definitions for Infection Specifically Adapted for Newborns. Journal of Hospital Infection, 61, 300-311. http://dx.doi.org/10.1016/j.jhin.2005.03.014

[15] Horan, T.C., Andrus, M. and Dudeck, M.A. (2008) CDC/NHSN Surveillance Definition of Health Care-Associated Infection and Criteria for Specific Types of Infections in the Acute Care Setting. American Journal of Infection Control, 36, 309-332.

[16] Nash, P. (2006) Umbilical Catheters, Placement, and Complication Management. Journal of Infusion Nursing, 29, 346352.

[17] Antunes, L.C., Rugolo, L.M. and Crocci, A.J. (2003) Effect of Preterm Infant Position on Weaning from Mechanical Ventilation. The Journal of Pediatrics, 79, 239-244.

[18] Kabra, N.S., Kumar, M. and Shah, S.S. (2005) Multiple versus Single Lumen Umbilical Venous Catheter for Newborn Infants. Cochrane Database of Systematic Reviews, 20, Article ID: CD004498.

[19] Mahieu, L.M., de Muynck, A.O., Leven, M.M., de Dooy, J.J., Goossens, H.J. and Van Reempts, P.J. (2001) Risk Factors for Central Vascular Catheter-Associated Bloodstream Infections among Patients in a Neonatal Intensive Care Unit. Journal of Hospital Infection, 48, 108-116. http://dx.doi.org/10.1053/jhin.2001.0984

[20] Schwab, F., Geffers, C., Barwolff, S., Ruden, H. and Gastmeier, P. (2007) Reducing Neonatal Nosocomial Bloodstream Infections through Participation in a National Surveillance System. Journal of Hospital Infection, 65, 319-325.

[21] Schulman, J., Stricof, R., Stevens, T.P., Horgan, M., Gase, K., Holzman, I.R., Koppel, R.I., Nafday, S., Gibbs, K., Angert, R., Simmond, S.A., Furdon, S.A. and Saiman, L., New York State Regional Perinatal Care Centres (2011) Statewide NICU Central-Line-Associated Bloodstream Infection Rates Decline after Bundles and Checklists. Pediatrics, 127, 436-444.

[22] Chien, L., Macnab, Y., Aziz, K., Andrews, W., McMillan, D. and Lee, S. (2002) The Canadian Network Variations in Central Venous Catheter-Related Infections Risk among Canadian Neonatal Intensive Care Units. Pediatric Infection 
Disease Journal, 21, 505-511. http://dx.doi.org/10.1097/00006454-200206000-00006

[23] Arnts, J.J.A., Bullens, L.M., Groenewoud, J.M.M. and Liem, K.L. (2014) Comparison of Complication Rates between Umbilical and Peripherally Inserted Central Venouscatheters in Newborns. Journal of Obstetric, Gynecologic, \& Neonatal Nursing, 43, 205-215. 
Scientific Research Publishing (SCIRP) is one of the largest Open Access journal publishers. It is currently publishing more than 200 open access, online, peer-reviewed journals covering a wide range of academic disciplines. SCIRP serves the worldwide academic communities and contributes to the progress and application of science with its publication.

Other selected journals from SCIRP are listed as below. Submit your manuscript to us via either submit@scirp.org or Online Submission Portal.
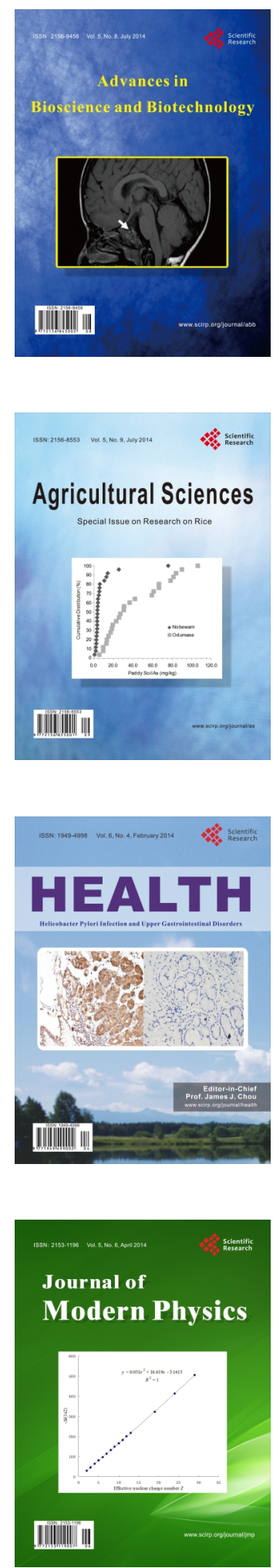
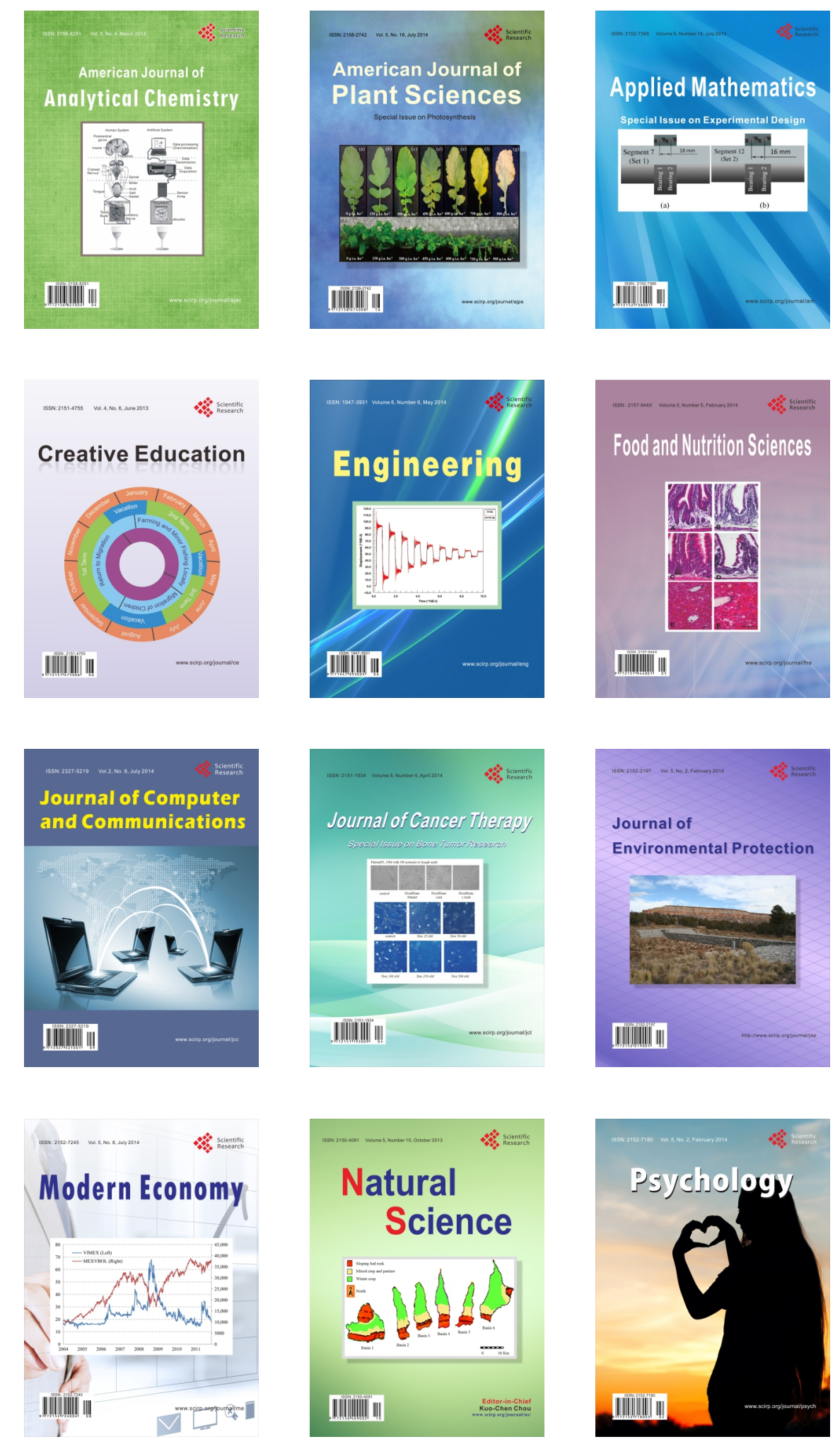